\title{
Postoperative Spinal Epidural Hematoma: The Danger Caused by the Misuse of Thrombin- Containing Local Hemostatics
}

\author{
Dong Ki Ahn, Won Shik Shin, Go We Kim, Ki Hyuk Koo \\ Department of Orthopedic Surgery, Seoul Sacred Heart General Hospital, Seoul, Korea
}

\begin{abstract}
Study Design: Retrospective case-control study.
Purpose: To examine the hypothesis that the misuse of thrombin-containing local hemostatics (TCLH) increases the risk of postoperative spinal epidural hematoma (POSEH).

Overview of Literature: Many studies have focused on hypocoagulability as a risk factor for POSEH. However, there are no prior reports on the increased risk of POSEH in hypercoagulable states.

Methods: Posterior instrumented lumbar spine surgery cases over 2 consecutive years were divided into two groups: a study group (98 patients in whom TCLH was used) and a control group (176 patients in whom TCLH was not used). The excess TCLH matrix that was not associated with blood clot was not removed from the patients in the study group. The senior author decided whether to use TCLH or not. Suction drains were used in all patients. The demographics, coagulation-related factors, and intraoperative factors of the patients in the two groups were analyzed. The development of POSEH was compared between the two groups.

Results: The two groups were homogenous in demographics (age and sex), coagulation-related factors (platelet count, prothrombin time, activated partial thromboplastin time, and platelet function analysis), and surgical factors (total blood loss, operation time, blood loss/10 minutes, number of fusion segments, posterolateral fusion/posterior lumbar interbody fusion, and virgin or revision surgery). POSEH developed more frequently in the patients in the study group than in those in the control group (14/98 patients, 14.3\% vs. $3 / 176$ patients, $1.7 \%$, respectively; $p=0.001$; odds ratio, 17.1 .

Conclusions: TCLH causes blood clot not only at the edge of damaged vessels but also at the site of extravascular blood. Excess TCLH matrix not associated with blood clot at the epidural space can enhance POSEH development because early clotted hematomas do not drain through suction drains.
\end{abstract}

Keywords: Postoperative spinal epidural hematoma; Thrombin-containing local hemostatics

\section{Introduction}

Although the incidence of postoperative spinal epidural hematoma (POSEH) is not high, it is considered a major complication, potentially leading to serious neurologi- cal deficit and requiring prompt surgical treatment [1-3]. While spontaneous or post-intervention spinal epidural hematomas have a close relationship with coagulopathy and antithrombotic or antiplatelet therapy, postoperative cases of spinal epidural hematoma have not been proven

Received Feb 11, 2017; Revised Mar 16, 2017; Accepted Apr 10, 2017

Corresponding author: Won Shik Shin

Department of Orthopedic Surgery, Seoul Sacred Heart General Hospital, 259 Wangsan-ro, Dongdaemun-gu, Seoul 02488, Korea

Tel: +82-2-966-1616, Fax: +82-2-968-2394, E-mail: dr.wonshik@hanmail.net 
to be related to such factors $[4,5]$. On the contrary, hypercoagulability or early clotting due to local hemostatics is presumed to disrupt the proper functioning of suction drains and leave larger hematomas at the surgical site. Local hemostatics that contain thrombin have been used since their approval by Food and Drug Administration in 1999. The user manual recommends removing excess hemostatic matrix after hemostasis is achieved. However, there are no studies showing the consequences of incomplete removal of thrombin, especially how this influences the formation of POSEH. We observed that POSEH occurred more frequently when using thrombin-containing local hemostatic (TCLH). Thrombin enhances not only fibrin clot formation at the bleeding site but also induces large clotted extravascular hematoma formation that cannot be removed through suction drains. Thus, in this study, we hypothesized that retained excess TCLH matrix unassociated with blood clot can lead to symptomatic POSEH.

\section{Materials and Methods}

This was a retrospective, observational study. TCLH (FloSeal, Baxter, Deerfield, IL, USA) was selectively used at our institution for instrumented spine surgeries between January 2013 and December 2014. All posterior instrumented spine surgeries during this period were retrospectively reviewed. Patients who did not undergo epidural space exposure by laminectomy and those who underwent minimally invasive surgeries were excluded. Initially, 438 patients were included. Among them, 98 patients in whom TCLH was used were classified as the study group; the remaining 340 patients in whom TCLH was not used were classified as the control group. The decision to use TCLH at the time of surgery was arbitrarily made by the senior author. Therefore, the two groups were not homogenous in surgical magnitude; 164 patients in the control group with a shorter operation time than average were excluded. Therefore, in total, the control group consisted of 176 patients. If a patient underwent multiple surgeries, each surgery was counted as a different case. The type of surgery was classified as posterolateral fusion (PLF) or posterior lumbar interbody fusion (PLIF). Patients who underwent both types of surgery were included in PLIF.

We did not remove the excess TCLH matrix after clot formation. Furthermore, we added the remaining TCLH

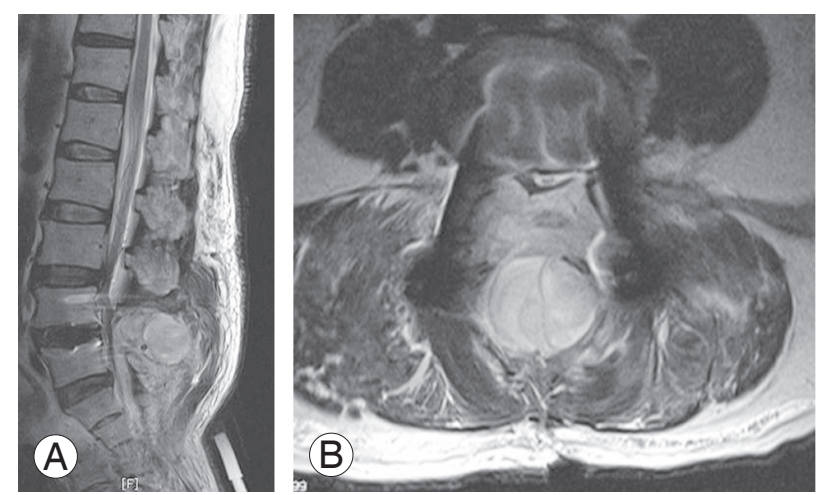

Fig. 1. Postoperative spinal epidural hematoma was diagnosed using magnetic resonance imaging. (A) T2-weighted sagittal image. (B) T2weighted axial image that showed an epidural heterogeneous round mass compressing the thecal sac.

in the epidural space at the conclusion of the surgery. All surgeries were performed by the senior author through a standard posterior approach, and two suction drains were used for every patient. Each drain had a 1.6-mm diameter and was connected to a negative-pressure bag with a pressure of $120 \pm 30 \mathrm{~mm} \mathrm{Hg}$ (Ez-VAC, EZ Medisys, Koyang, Korea). Suction drains were removed on postoperative day 3. For comparing the homogeneity between the two groups, demographic, surgical, and coagulation-related parameters were analyzed. A revision surgery was defined as a repeated surgery at the same segments or adjacent segments. POSEH was postoperatively identified by the presence of new neurological symptoms and characteristic findings on magnetic resonance imaging (MRI) (Fig. 1) and finally confirmed by improvement after surgical removal. For statistical analysis, independent t-tests and chi-square tests were used for comparing the homogeneity for parametric and non-parametric variables, respectively. The Levene's test was used for analyzing the equality of variances. Odds ratio (OR) was determined using the Fisher's exact test. Because there were some heterogeneous variables, the independence of TCLH as a risk factor was confirmed using multivariable logistic regression analysis. SPSS for Windows software package (ver. 16.0; SPSS Inc., Chicago, IL, USA) was used.

\section{Results}

Demographics and coagulation-related factors are presented in Table 1. There was no difference between the two groups for all factors. Surgical factors are presented in Table 2; there were no significant differences between the 
Table 1. Demographics and coagulation related factors

\begin{tabular}{lcccccccc} 
Group & Cases & Age $(\mathrm{yr})$ & Sex (male/female) & NFS & PFA & Platelet (/mL) & PT & aPTT \\
Study & 98 & 67.1 & $32 / 66$ & 1.7 & 164.3 & 240,500 & 10.5 & 28.2 \\
Control & 176 & 67.4 & $58 / 118$ & 1.5 & 214.3 & 241,926 & 10.4 & 28.4 \\
\hline$p$-value & - & 0.781 & - & 0.153 & 0.117 & 0.834 & 0.223 & 0.50 \\
\hline
\end{tabular}

NFS, number of fusion segments; PFA, platelet function analysis-epinephrine; PT, prothrombin time; aPTT, activated partial thromboplastin time.

Table 2. Intraoperative factors and postoperative spinal epidural hematoma

\begin{tabular}{lcccccc} 
Group & TBL $(\mathrm{mL})$ & OPT $(\mathrm{min})$ & $\mathrm{BL} / 10 \mathrm{~min}(\mathrm{~mL})$ & PLF/PLIF & V/R & POSEH (\%) \\
Study & 1,255 & 198.8 & 60.6 & $15 / 83$ & $62 / 36$ & $14 / 98(14.3)$ \\
Control & 1,145 & 189.3 & 60.3 & $33 / 143$ & $116 / 60$ & $3 / 176(1.4)$ \\
\hline$p$-value & 0.166 & 0.107 & 0.913 & 0.511 & 0.693 & 0.001 (odds ratio=17.1) \\
\hline
\end{tabular}

TBL, total blood loss; OPT, operation time; BL/10 min, blood loss per 10 minutes; PLF/PLIF, posterolateral fusion/posterior lumbar interbody fusion; V/R, virgin/revision; POSEH, postoperative spinal epidural hematoma.

groups. POSEH developed in $14 / 98$ patients (14.3\%) in the study group and $3 / 176$ patients (1.4\%) in the control group. There was a significant difference between the two groups in terms of POSEH (OR, 17.1).

\section{Discussion}

One of the most feared complications of spine surgery is neurological injury. Occasionally, this can be prevented using proactive surgical maneuvers. According to Cramer et al. [6], the most common cause of neurological injury after spine surgery is POSEH. Unfortunately, little is known about POSEH. Studies indicate that symptomatic POSEH should be surgically removed as soon as possible to prevent permanent neurological sequelae [1-3]. Furthermore, a consensus has not been reached regarding the general incidence, pathogenesis, type and degree of neurological symptoms, and risk factors [7-11]. Therefore, preventive strategies are limited with the exception of suction drain implementation. Previous studies have shown that suction drains reduce the remaining epidural hematoma [12], and reducing hematomas by using drains is sometimes related to symptom development $[11,13]$ and sometimes not [14-17]. However, most previous studies showthat POSEH was not prevented by suction drains [3,18-23].

Hemostasis is mechanistically divided into two steps. First, a damaged vessel contracts and platelets aggregate and plug the damaged area. Then, Von Willebrand factors released from the endothelial cells enhance platelet aggregation. This is referred to as primary hemostasis. Subsequently, activated clotting factors in the plasma activate thrombin. Fibrinogen is then converted into fibrin by thrombin. Finally, fibrin mesh reinforces the aggregated platelet plug. This is referred to as secondary hemostasis [24]. Meanwhile, extravascular blood has no vascular factors and mainly depends on secondary hemostasis to form a clot. We presume that unclotted blood easily drains through suction drains. If blood in the epidural space is clotted before suction drains begin to work, the amount of remaining hematoma would increase. The factors that retard the vacuum state of epidural space are multilevel, revision, and instrumented surgeries. These are the currently suspected risk factors of POSEH $[9,13,25]$. Although we reform the closed vacuum state of the epidural space as soon as possible, if extravascular blood clots faster than usual, the amount of remaining hematoma would also increase. Theoretically, TCLH can accelerate the clotting of extravascular blood. Although the user manual of FloSeal recommends removing hemostatic matrix that is not associated with the clot, there is no warning about the risk of POSEH. Before we began the present study, we performed a simple preliminary experiment wherein we added TLCH into blood, and delayed activation of suction resulted in improper function of suction drains. We then performed the present study to evaluate this further in real-time clinical scenarios. TCLH dramatically increased symptomatic POSEH (OR, 14). It should be noted that we did not expressly follow the manufacturer's guidelines, and the excess TLCH matrix was 
not removed. Furthermore, we added TCLH to the epidural space to stop the bleeding and left it until just prior to wound closure. Doing this may increase the incidence of POSEH. We strongly believe that unused active thrombin should be removed to prevent unexpected early clotting of extravascular blood before appropriate drainage. Previous studies that indicated multilevel surgery and revision surgery as risk factors of POSEH did not specify the reasons $[9,13,25]$. There would be many reasons for that; however, we thought it as a cardinal one that both conditions delayed activation of suction drains because of loss of tissue elasticity and longer wound closing time.

Emori et al. [26] reported an essential thrombocythemia case that demonstrated a link between hypercoagulability and POSEH. They did not, however, clarify the mechanism underlying this link. We believe that we have presented a hypothesis linking hypercoagulability to POSEH. Although we cannot explain the overall pathogenetic mechanisms with this model, we can say that hypercoagulability or iatrogenic enhancement of the clotting of extravascular blood increases the possibility of POSEH. Some studies claim that antiplatelet medication is one of the risk factors for POSEH $[5,8]$. Decreased platelet function increases bleeding from a damaged vessel because platelet plugging is inhibited. However, the clotting time of extravascular blood would not be influenced. The likelihood of POSEH, here, would theoretically increase. If the clotting time of extravascular blood is also prolonged, the remaining blood can be easily drained through suction drains. Based on the above, we believe that POSEH can be prevented by the timely activation of suction drains prior to the clotting of extravascular blood.

There are limitations of the present study. Because this was not a prospective study and the decision whether to use TCLH was arbitrarily determined, the two groups were not completely homogenous. For stratified sampling, the short time operations in non-TCLH cases were excluded from the control group. The results of this study should be cautiously interpreted as we did not remove excess TCLH matrix after achieving hemostasis. It remains unclear whether TCLH still increases the risk of POSEH even after completely removing the excess matrix. However, OR of the study group was high enough to be meaningful. To the best of our knowledge, our study is the first that presents one of possible pathogenesis model of POSEH. Though this is not a prospective study, we believe it serves as a baseline for future studies on this complex disease.

\section{Conclusions}

TCLH forms blood clots not only at the edge of damaged vessels but also at the site of extravascular blood. The remaining excess TCLH matrix at the epidural space can lead to the development of POSEH because early clotted hematoma does not drain through suction drains. Excess TCLH matrix must be completely removed as it accelerates the clotting of extravascular blood in the epidural space before suction drains get activated. The potential hazardous influence of TCLH should be clarified in the user manual.

\section{Conflict of Interest}

No potential conflict of interest relevant to this article was reported.

\section{References}

1. Amiri AR, Fouyas IP, Cro S, Casey AT. Postoperative spinal epidural hematoma (SEH): incidence, risk factors, onset, and management. Spine J 2013;13:134-40.

2. Cabana F, Pointillart V, Vital J, Senegas J. Postoperative compressive spinal epidural hematomas. 15 cases and a review of the literature. Rev Chir Orthop Reparatrice Appar Mot 2000;86:335-45.

3. Yi S, Yoon DH, Kim KN, Kim SH, Shin HC. Postoperative spinal epidural hematoma: risk factor and clinical outcome. Yonsei Med J 2006;47:326-32.

4. Cuellar JM, Petrizzo A, Vaswani R, Goldstein JA, Bendo JA. Does aspirin administration increase perioperative morbidity in patients with cardiac stents undergoing spinal surgery? Spine (Phila Pa 1976) 2015;40:629-35.

5. Korinth MC, Gilsbach JM, Weinzierl MR. Lowdose aspirin before spinal surgery: results of a survey among neurosurgeons in Germany. Eur Spine J 2007;16:365-72.

6. Cramer DE, Maher PC, Pettigrew DB, Kuntz Ct. Major neurologic deficit immediately after adult spinal surgery: incidence and etiology over 10 years at a single training institution. J Spinal Disord Tech 2009;22:565-70.

7. Awad JN, Kebaish KM, Donigan J, Cohen DB, Kos- 
tuik JP. Analysis of the risk factors for the development of post-operative spinal epidural haematoma. J Bone Joint Surg Br 2005;87:1248-52.

8. Kebaish KM, Awad JN. Spinal epidural hematoma causing acute cauda equina syndrome. Neurosurg Focus 2004;16:e1.

9. Kou J, Fischgrund J, Biddinger A, Herkowitz H. Risk factors for spinal epidural hematoma after spinal surgery. Spine (Phila Pa 1976) 2002;27:1670-3.

10. Lawton MT, Porter RW, Heiserman JE, Jacobowitz R, Sonntag VK, Dickman CA. Surgical management of spinal epidural hematoma: relationship between surgical timing and neurological outcome. J Neurosurg 1995;83:1-7.

11. Leonardi MA, Zanetti M, Saupe N, Min K. Early postoperative MRI in detecting hematoma and dural compression after lumbar spinal decompression: prospective study of asymptomatic patients in comparison to patients requiring surgical revision. Eur Spine J 2010;19:2216-22.

12. Mirzai H, Eminoglu M, Orguc S. Are drains useful for lumbar disc surgery? A prospective, randomized clinical study. J Spinal Disord Tech 2006;19:171-7.

13. Sokolowski MJ, Garvey TA, Perl J 2nd, et al. Prospective study of postoperative lumbar epidural hematoma: incidence and risk factors. Spine (Phila Pa 1976) 2008;33:108-13.

14. Awwad EE, Smith KR Jr. MRI of marked dural sac compression by surgicel in the immediately postoperative period after uncomplicated lumbar laminectomy. J Comput Assist Tomogr 1999;23:969-75.

15. Kotilainen E, Alanen A, Erkintalo M, Helenius H, Valtonen S. Postoperative hematomas after successful lumbar microdiscectomy or percutaneous nucleotomy: a magnetic resonance imaging study. Surg Neurol 1994;41:98-105.

16. Djukic S, Vahlensieck M, Resendes M, Genant HK. The lumbar spine: postoperative magnetic resonance imaging. Bildgebung 1992;59:136-46.

17. Ikuta K, Tono O, Tanaka T, et al. Evaluation of postoperative spinal epidural hematoma after microendoscopic posterior decompression for lumbar spinal stenosis: a clinical and magnetic resonance imaging study. J Neurosurg Spine 2006;5:404-9.

18. Brown MD, Brookfield KF. A randomized study of closed wound suction drainage for extensive lumbar spine surgery. Spine (Phila Pa 1976) 2004;29:1066-8.

19. Chimenti P, Molinari R. Post-operative spinal epidural hematoma causing American Spinal Injury Association B spinal cord injury in patients with suction wound drains. J Spinal Cord Med 2013;36:213-9.

20. Kanayama M, Oha F, Togawa D, Shigenobu K, Hashimoto T. Is closed-suction drainage necessary for single-level lumbar decompression?: review of 560 cases. Clin Orthop Relat Res 2010;468:2690-4.

21. Parker MJ, Livingstone V, Clifton R, McKee A. Closed suction surgical wound drainage after orthopaedic surgery. Cochrane Database Syst Rev 2007;(3):CD001825.

22. Scuderi GJ, Brusovanik GV, Fitzhenry LN, Vaccaro AR. Is wound drainage necessary after lumbar spinal fusion surgery? Med Sci Monit 2005;11:CR64-6.

23. Walid MS, Abbara M, Tolaymat A, et al. The role of drains in lumbar spine fusion. World Neurosurg 2012;77:564-8.

24. Li Z, Delaney MK, O’Brien KA, Du X. Signaling during platelet adhesion and activation. Arterioscler Thromb Vasc Biol 2010;30:2341-9.

25. Uribe J, Moza K, Jimenez O, Green B, Levi AD. Delayed postoperative spinal epidural hematomas. Spine J 2003;3:125-9.

26. Emori M, Takebayashi T, Imoto K, Ueno S, Mizuno S, Yamashita T. Spinal surgery in a patient with essential thrombocythemia resulting in leg paraplegia: a case report. Spine J 2013;13:e7-10. 